\title{
Modeling invasive lobular breast carcinoma by CRISPR/Cas9-mediated somatic genome editing of the mammary gland
}

\author{
Stefano Annunziato, ${ }^{1,6}$ Sjors M. Kas,${ }^{1,6}$ Micha Nethe, ${ }^{1}$ Hatice Yücel, ${ }^{1}$ Jessica Del Bravo, ${ }^{2}$ \\ Colin Pritchard, ${ }^{2}$ Rahmen Bin Ali, ${ }^{2}$ Bas van Gerwen, ${ }^{3}$ Bjørn Siteur, ${ }^{3}$ Anne Paulien Drenth, ${ }^{1}$ Eva Schut, ${ }^{1}$ \\ Marieke van de Ven, ${ }^{3}$ Mirjam C. Boelens, ${ }^{1}$ Sjoerd Klarenbeek, ${ }^{4}$ Ivo J. Huijbers, ${ }^{2}$ \\ Martine H. van Miltenburg, ${ }^{1}$ and Jos Jonkers ${ }^{1,5}$ \\ ${ }^{1}$ Division of Molecular Pathology, The Netherlands Cancer Institute, 1066 CX Amsterdam, The Netherlands; ${ }^{2}$ Transgenic Core \\ Facility, Mouse Clinic for Cancer and Aging (MCCA), The Netherlands Cancer Institute, 1066 CX Amsterdam, The Netherlands; \\ ${ }^{3}$ Preclinical Intervention Unit, Mouse Clinic for Cancer and Aging (MCCA), The Netherlands Cancer Institute, 1066 CX \\ Amsterdam, The Netherlands; ${ }^{4}$ Experimental Animal Pathology, The Netherlands Cancer Institute, 1066 CX Amsterdam, The \\ Netherlands; ${ }^{5}$ Cancer Genomics Netherlands, The Netherlands Cancer Institute, 1066 CX Amsterdam, The Netherlands
}

Large-scale sequencing studies are rapidly identifying putative oncogenic mutations in human tumors. However, discrimination between passenger and driver events in tumorigenesis remains challenging and requires in vivo validation studies in reliable animal models of human cancer. In this study, we describe a novel strategy for in vivo validation of candidate tumor suppressors implicated in invasive lobular breast carcinoma (ILC), which is hallmarked by loss of the cell-cell adhesion molecule E-cadherin. We describe an approach to model ILC by intraductal injection of lentiviral vectors encoding Cre recombinase, the CRISPR/Cas9 system, or both in female mice carrying conditional alleles of the $C d h 1$ gene, encoding for E-cadherin. Using this approach, we were able to target ILC-initiating cells and induce specific gene disruption of Pten by CRISPR/Cas9-mediated somatic gene editing. Whereas intraductal injection of Cas9-encoding lentiviruses induced Cas9-specific immune responses and development of tumors that did not resemble ILC, lentiviral delivery of a Pten targeting single-guide RNA (sgRNA) in mice with mammary gland-specific loss of E-cadherin and expression of Cas9 efficiently induced ILC development. This versatile platform can be used for rapid in vivo testing of putative tumor suppressor genes implicated in ILC, providing new opportunities for modeling invasive lobular breast carcinoma in mice.

[Keywords: somatic gene editing; breast cancer; invasive lobular carcinoma; CRISPR/Cas9; intraductal injection; mouse models]

Supplemental material is available for this article.

Received February 10, 2016; accepted in revised form May 27, 2016.

Invasive lobular carcinoma (ILC) is the second most common type of human breast cancer, accounting for $8 \%-$ $14 \%$ of all breast cancer cases (Martinez and Azzopardi 1979; Borst and Ingold 1993; Wong et al. 2014). It is characterized by discohesive epithelial cells infiltrating the surrounding tissue in single-file patterns accompanied by an abundant presence of fibroblasts and collagen depo-

${ }^{6}$ These authors contributed equally to this work.

Corresponding authors: j.jonkers@nki.nl, m.v.miltenburg@nki.nl Article is online at http://www.genesdev.org/cgi/doi/10.1101/gad.279190. 116. sition. The majority of human ILCs shows loss of the cellcell adhesion protein E-cadherin due to inactivating mutations, loss of heterozygosity $(\mathrm{LOH})$, and methylation of the CDH1 gene promoter (Moll et al. 1993; Vos et al. 1997; Droufakou et al. 2001; Ciriello et al. 2015) or impaired integrity of the E-cadherin-catenin membrane

\footnotetext{
(C) 2016 Annunziato et al. This article is distributed exclusively by Cold Spring Harbor Laboratory Press for the first six months after the full-issue publication date (see http://genesdev.cshlp.org/site/misc/terms.xhtml). After six months, it is available under a Creative Commons License (Attribution-NonCommercial 4.0 International), as described at http://creativecommons.org/licenses/by-nc/4.0/.
} 
complex (Rakha et al. 2010). Intriguingly, mice with tissue-specific loss of E-cadherin in mammary epithelial cells do not develop mammary tumors (Boussadia et al. 2002; Derksen et al. 2006, 2011). It has been shown that E-cadherin loss in mammary epithelial cells leads to apoptosis (Boussadia et al. 2002). However, multifocal ILC development is induced by combined (mammary) epithelium-specific loss of E-cadherin and p53 (Derksen et al. 2006, 2011) or E-cadherin and PTEN (MC Boelens, M Nethe, S Klarenbeek, E Schut, JR de Ruiter, N Bonzanni, AL Zeeman, E Wientjens, E van der Burg, L Wessels, et al., in prep.), highlighting the importance of co-occurring mutations in ILC development.

Recent studies have shed light on the mutational landscape of human ILC, showing that CDH1 mutations are accompanied by alterations in a plethora of additional genes, of which only a few have been mechanistically linked to ILC formation or tumorigenesis in general (Ciriello et al. 2015). Discrimination between passenger mutations and bona fide driver events has become an urgent priority that requires well-designed validation studies in model systems. A gene-by-gene approach can have several bottlenecks, especially when in vivo mouse models with complex genotypes have to be generated. Forward genetic approaches in E-cadherin-deficient mouse models can help disentangle this complexity, but promising "hits" from screens ultimately need ad hoc validation experiments.

For these reasons, new technologies are needed to expand the genetic toolbox of cancer biologists and allow a more rapid and systematic in vivo interrogation of gene perturbations. In this regard, the advent of CRISPR/Cas9 technologies for somatic genome editing has already paved the way for a new generation of nongermline animal tumor models. For example, liver-specific gene disruption was achieved by transient delivery of components of the CRISPR/Cas9 system in the tail veins of mice, leading to hepatocellular carcinoma (Xue et al. 2014; Weber et al. 2015). Similar approaches have been used to deliver targeted oncogenic mutations to the lung (Platt et al. 2014; Sánchez-Rivera et al. 2014), brain (Zuckermann et al. 2015), and pancreas (Chiou et al. 2015).

Here we describe a novel approach to model ILC by delivering lentiviral vectors to the adult mammary gland by intraductal injection. We show that administration of Cre-encoding lentiviruses results in sporadic targeting of mammary epithelial cells and initiation of multifocal tumor development in mice harboring-together with conditional Cdh1 alleles-a conditional activating AktE17K mutation or conditional Pten alleles. Furthermore, we implemented CRISPR/Cas9-mediated somatic gene editing in mammary tissue and, as a proof of concept, inactivated PTEN expression in E-cadherin-deficient mammary epithelial cells. However, somatic delivery of Cas9 resulted in mammary tumors that did not resemble ILC and showed strong immune infiltration, which is most likely due to previously reported Cas9-specific immune responses (Wang et al. 2015). In contrast, intraductal injection of lentiviruses encoding a single-guide RNA (sgRNA) targeting Pten in female mice with mam- mary-specific loss of E-cadherin and expression of Cas9 endonuclease from a conditional knock-in allele resulted in ILC formation without a massive influx of immune cells. Collectively, we describe a platform that can be used for rapid in vivo validation of candidate tumor suppressors implicated in ILC and the development of novel mouse models of this breast cancer subtype.

\section{Results}

Transduction of ductal epithelial cells by intraductal injection of lentiviral Cre

Site-specific delivery of adenoviral or lentiviral Cre has been successfully used in several conditional mouse models to initiate tumor formation in different tissues, including the lung, the liver, muscle, and the pancreas (Meuwissen et al. 2001; Harada et al. 2004; Kirsch et al. 2007; Chiou et al. 2015). In our study, we set out to implement intraductal injections of lentiviral vectors as a tool to achieve mammary gland-specific Cre expression and/ or CRISPR/Cas9-mediated genome editing. In the past, intraductal injection of Cre-encoding adenoviruses was successfully used to activate expression of oncogenic fusion genes in the mammary gland of genetically engineered mice, leading to mammary tumors (Rutkowski et al. 2014; Tao et al. 2014). To verify the applicability of this technique for intraductal delivery of lentiviruses, we performed injections of female virgin FVB mice with a lentiviral vector expressing GFP $(n=8)$, revealing efficient transduction of the ductal tree (Fig. 1A; Supplemental Fig. S1A,B). To confirm that lentiviral delivery of Cre was able to recombine conditional alleles in vivo, we performed injection of a Cre-encoding lentiviral vector (Lenti-Cre) into double-fluorescent $\mathrm{mT} / \mathrm{mG}$ Cre reporter mice $(n=8)$ in which membrane targeted GFP (mGFP) was expressed after Cre-mediated excision of mTomato (Muzumdar et al. 2007). GFP-positive cells were observed throughout the ductal trees of mammary glands from $\mathrm{mT} / \mathrm{mG}$ mice injected with Lenti-Cre (Fig. 1B). Immunostaining of mammary gland sections with an anti-GFP antibody showed extensive GFP labeling in both luminal and basal cells of the ductal epithelium at $2 \mathrm{wk}$ after injection (Fig. 1C,D). Similar results were observed with intraductal injection of Adeno-Cre in $m T / m G$ mice (Supplemental Fig. S1C,D). These data demonstrate that intraductal injection of Cre-encoding lentiviruses induces efficient in vivo recombination of conditional alleles in mammary epithelium, as previously shown by other groups using Adeno-Cre (Russell et al. 2003; Rutkowski et al. 2014; Tao et al. 2014).

\section{Intraductal injection of Lenti-Cre promotes ILC formation in mice carrying conditional alleles of ILC drivers}

Next, we investigated whether intraductal injections could be used to initiate mammary tumor formation in genetically engineered mice carrying conditional alleles of genes implicated in human ILC. For this purpose, 


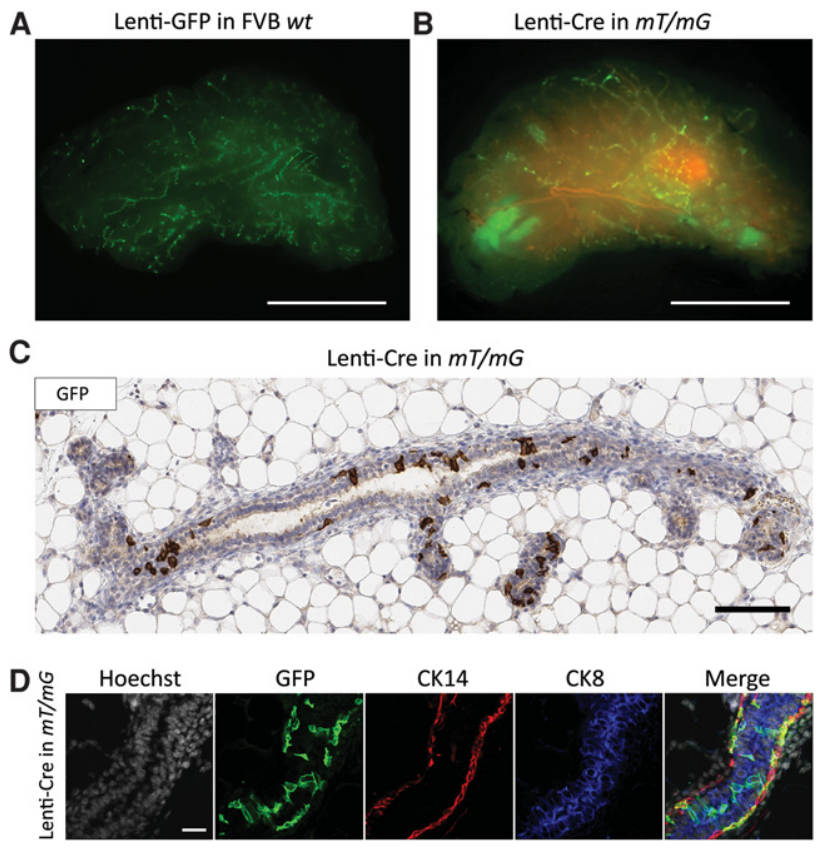

Figure 1. Intraductal injection of Lenti-Cre allows in vivo recombination in the mammary epithelium. (A) Fluorescence microscopy of GFP expression in a representative whole-mount mammary gland after intraductal injection of Lenti-GFP in FVB wild-type animals. $n=8$. Mice were analyzed $14 \mathrm{~d}$ after injection. Bar, $5 \mathrm{~mm}$. (B) Fluorescence microscopy of GFP expression in a representative whole-mount mammary gland after intraductal injection of Lenti-Cre in $m T / m G$ Cre reporter mice. $n=8$. Mice were analyzed $14 \mathrm{~d}$ after injection. Bar, $5 \mathrm{~mm}$. (C) Immunohistochemical detection of GFP expression in a representative mammary gland section from $\mathrm{mT} / \mathrm{mG}$ Cre reporter mice intraductally injected with Lenti-Cre. Bar, $100 \mu \mathrm{m}$. $(D)$ Immunofluorescence analysis of mammary gland sections from Lenti-Cre-injected $\mathrm{mT} / \mathrm{m}$ GCre reporter mice showing GFP expression in cytokeratin 8 (CK8)-positive and CK14-positive cells. Bar, $25 \mu \mathrm{m}$.

we developed a genetically engineered mouse model (GEMM) in which transgenic Cre expression under transcriptional control of the Wap gene promoter induces mammary gland-specific inactivation of E-cadherin and activation of the oncogenic AKT-E17K isoform. These mice were generated by introduction of a Cre conditional invCAG-AktE17K-IRES-Luc allele into the Colla1 locus of embryonic stem cells (ESCs) derived from WapCre;Cdh1 $1^{F / F}$ mice and subsequent production of chimeric mice by blastocyst injection of the modified ESCs (Supplemental Fig. S2A,B; Huijbers et al. 2014). High-quality male chimeras were mated with $C d h 1^{F / F}$ females to generate a cohort of WapCre;Cdh1 $1^{F / F}$; Col1a1 ${ }^{\text {invCAG-AktE17K-IRES-Luc/+ }}$ (WapCre;Cdh1 ${ }^{\text {F/F }}$;Akt$E 17 K)$ female mice $(n=15)$, which were monitored for spontaneous tumor development. In parallel, WapCrenegative $C d h 1^{F / F}$;Colla1 invCAG-AktE17K-IRES-Luc/+ $\mid C d h 1^{F / F}$; Akt-E17K) female mice $(n=7)$ were used for intraductal injections with Lenti-Cre (Fig. 2A). All WapCre;Cdh1 $1^{F / F}$; Akt-E17K female mice developed multifocal ILC lesions in all mammary glands due to concomitant inactivation of E-cadherin and expression of the oncogenic AKT-E17K variant accompanied by luciferase expression (Fig. 2B). Likewise, intraductal injection of Lenti-Cre into female $C d h 1^{F / F}$;Akt-E17K mice resulted in specific bioluminescence signals building up over time (Fig. 2C; Supplemental Fig. S3A). Following sacrifice of the mice $\sim 30$ wk after injection (except for one mouse, which was sacrificed at $12 \mathrm{wk}$ with a palpable tumor), sectioning and hematoxylin and eosin $(\mathrm{H} \& \mathrm{E})$ staining of the injected mammary glands revealed multiple tumors in six out of seven injected glands (Supplemental Fig. S3B,C). Tumors showed a typical ILC histology with abundant collagen deposition and single files of cytokeratin 8 (CK8)-positive tumor cells infiltrating the surrounding tissue. Moreover, tumors showed recombined $C d h 1^{F}$ and invCAGAktE17K-IRES-Luc alleles (Supplemental Fig. S3D), were phospho-AKT ${ }^{\mathrm{Ser} 473}$-positive and E-cadherin-deficient, and were indistinguishable from those developing in the conventional WapCre;Cdh1 ${ }^{F / F} ; A k t-E 17 K$ model (Fig. 2D,E). To investigate whether local Lenti-Cre delivery could induce ILC formation driven by loss of tumor suppressor genes (TSGs) rather than activation of a potent oncogene such as Akt-E17K, we performed intraductal Lenti-Cre injections in $C d h 1^{F / F}$; Pten ${ }^{F / F}$ mice $(n=8)$ carrying conditional alleles of E-cadherin and the phosphatase and tensin homolog (Pten) gene, a negative regulator of the PI3K/AKT signaling pathway. Again, we observed multifocal ILC formation in seven out of eight injected mammary glands following sacrifice of the animals at 14 wk after injection. Tumors showed ILC histology, CK8-positive cells, and recombined $C d h 1^{F}$ and Pten ${ }^{F}$ alleles, resulting in loss of E-cadherin and PTEN (Fig. 2F; Supplemental Fig. S4A-D), similar to mammary tumors developing in the WapCre;Cdh1 ${ }^{F / F} ;$ Pten $^{F / F}$ mouse model (MC Boelens, M Nethe, S Klarenbeek, E Schut, JR de Ruiter, N Bonzanni, AL Zeeman, E Wientjens, E van der Burg, L Wessels, et al., in prep.). Mice injected with control lentiviruses (Lenti-GFP) did not show any ILC formation. Altogether, these data show that intraductal injection of Lenti-Cre in mice carrying conditional alleles of ILC driver genes targets mammary epithelial cells with ILC-initiating capacity, resulting in tumors that closely resemble their human counterparts.

\section{Intraductal injection of pSECC-sgPten in $\mathrm{Cdh} 1^{\mathrm{F} / \mathrm{F}}$ mice induces tumors that do not resemble ILC}

Having shown that ILC formation can be induced by intraductal injection of Lenti-Cre in $C d h 1^{F / F} ; A k t-E 17 K$ and $C d h 1^{F / F}{ } P_{t e n}{ }^{F / F}$ mice, we next explored the possibility of combining local Cre delivery with somatic gene editing by the CRISPR/Cas9 system to rapidly evaluate the contribution of candidate tumor suppressors to ILC formation in $C d h 1^{F / F}$ mice. For this approach, we used the pSECC vector, a lentiviral vector encoding Cre and the CRISPR components (a sgRNA targeting a gene of interest and the Streptococcus pyogenes Cas9) (SánchezRivera et al. 2014). pSECC vectors containing a nontargeting sgRNA (sgNT) or a validated sgRNA (sgPten) targeting the first exon of Pten were tested for their in vitro activity 
A

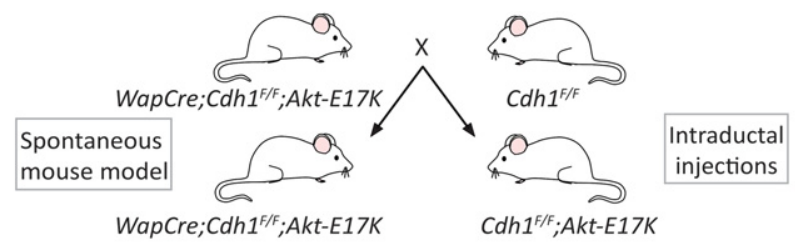

B

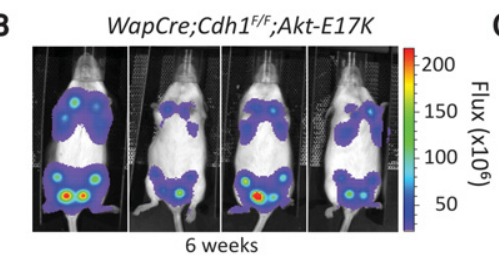

D

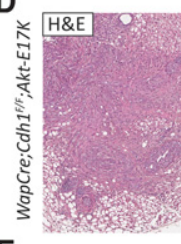

E-cadherin
Lenti-Cre in $C d h 1^{F / F} ; A k t-E 17 K$

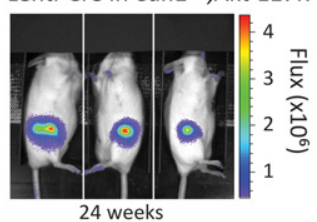

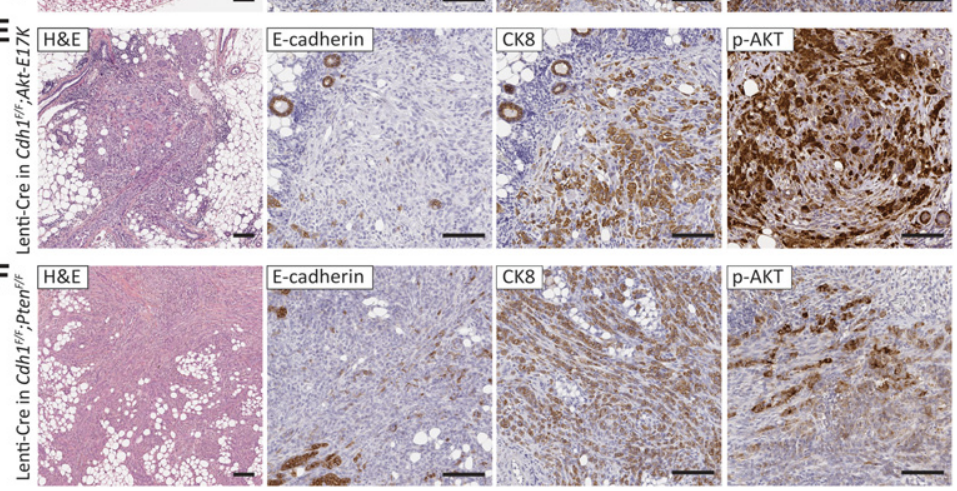

Figure 2. Intraductal injection of Lenti-Cre in $C d h 1^{F / F}$; Akt-E17K and $C d h 1^{F / F}{ }_{;}$Pten ${ }^{F / F}$ mice results in ILC formation. $(A)$ Breeding strategy for a matched comparison of ILC formation induced by transgenic WapCre expression or Lenti-Cre injection in $C d h 1^{F / F} ; A k t-E 17 K$ mice. (B) In vivo bioluminescence imaging of luciferase expression in WapCre; $\mathrm{Cdh}^{F / F}{ }$;Akt-E17K animals at 6 wk of age. $(C)$ In vivo bioluminescence imaging of luciferase expression in $C d h 1^{F / F}$;Akt-E17K mice 24 wk after intraductal injection of Lenti-Cre. $(D)$ Immunohistochemical analysis of E-cadherin, CK8, and phosphoAKT $^{\text {Ser473 }}$ expression in WapCre; Cdh1 $^{F / F} ;$; Akt-E17K (n $=15$ ) tumors. Tumors were analyzed at $6 \mathrm{wk}$ of age. Bars, $100 \mu \mathrm{m}$. (E) Immunohistochemical analysis of Ecadherin, CK8, and phospho-AKT ${ }^{\text {Ser473 }}$ expression in tumor sections from Lenti-Cre-injected $C d h 1^{F / F} ; A k t$ E17K animals. $n=7$. The tumor was analyzed $12 \mathrm{wk}$ after injection. Bars, $100 \mu \mathrm{m}$. $(F)$ Immunohistochemistry of E-cadherin, CK8, and phospho-AKT ${ }^{\text {er4473 }}$ in tumor sections from Lenti-Cre-injected $C d h 1^{F / F}{ }_{;}$Pten $^{F / F}$ animals. $n=8$. Tumors were analyzed $14 \mathrm{wk}$ after injection. Bars, $100 \mu \mathrm{m}$. in a Cre reporter cell line carrying a lox-stop-lox GFP cassette. GFP expression and Pten gene editing could be achieved efficiently and rapidly upon transduction of GFP reporter cells with the pSECC-sgPten vector (Fig. $3 \mathrm{~A}$; Supplemental Fig. S5A,B). To assess the in vivo recombination efficiency of these lentiviral vectors, we intraductally injected high-titer pSECC-sgNT into $\mathrm{mT} / \mathrm{mG}$ Cre reporter mice $(n=8)$. GFP staining of mammary glands at $2 \mathrm{wk}$ after injection confirmed GFP labeling of ductal epithelial cells (Supplemental Fig. S5C). Upon injection of pSECC-sgNT into $C d h 1^{F / F} ; A k t-E 17 K$ mice $(n=4)$, we observed bioluminescence signals building up in half (two out of four) of the injected mammary glands due to activation of the oncogenic Akt-E17K allele (Supplemental Fig. S5D). The luciferase-positive mammary glands showed tumors with typical ILC histology, expression of phospho-AKT ${ }^{\mathrm{Ser} 473}$ and CK8, and loss of Ecadherin, demonstrating that Cre expression from intraductally injected pSECC can give rise to ILC formation in predisposed mice (Fig. 3B; Supplemental Fig. S5E). We then sought to determine whether intraductal injection of pSECC-sgPten into $C d h 1^{F / F}$ mice $(n=48)$ was sufficient to induce ILC. As a control, $C d h 1^{F / F}$ mice were injected with pSECC-sgNT $(n=27)$. Following sacrifice of the mice $\sim 25$ wk after injection, we observed tumors in 12 out of $48 C d h 1^{F / F}$ mammary glands injected with pSECC-sgPten (Table 1). No lesions were observed in pSECC-sgNT-injected $C d h 1^{F / F}$ females. Notably, most tumors in pSECC-sgPten-injected Cdh1 $1^{F / F}$ female mice were not classified as ILCs and were composed of both E-cadherin-negative and E-cadherin-positive cells, indicating incomplete Cre-mediated recombination of the $C d h 1^{F}$ alleles (Fig. 3C,D, Supplemental Fig. S6A,B). The tumors were also strongly surrounded by infiltrating immune cells, which stained positive for CD4, CD8, and B220, indicating both T-cell and B-cell recruitment (Fig. 3C). It was shown previously that somatic expression of Cas9 in adult mice may trigger Cas9-specific immune responses (Wang et al. 2015). In an attempt to reduce immune recruitment, we tested whether transient immunosuppression by cyclosporin A administration (Howell et al. 1998; Meuwissen et al. 2001) would boost ILC development in pSECC-sgPten-injected $C d h 1^{F / F}$ females, but this was not the case (data not shown). We obtained genomic DNA from tumor-bearing mammary glands and confirmed target modification of Pten exon 1, resulting in frameshift mutations or larger deletions (Fig. 3E; Supplemental Fig. S6C). Indeed, immunofluorescence showed that tumors were PTEN-negative, resulting in activation of PI3K/AKT signaling (Supplemental Fig. 
A

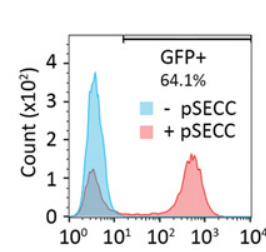

pSECC-sgPten in vitro
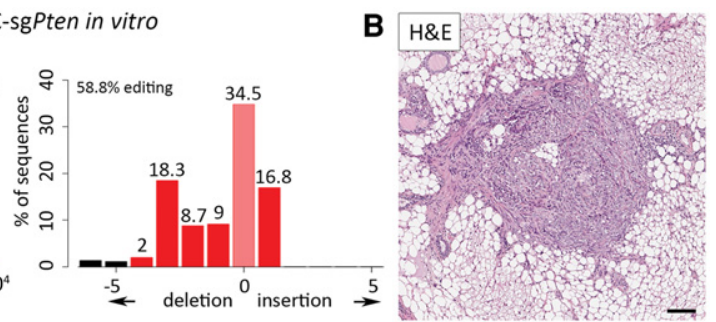

C

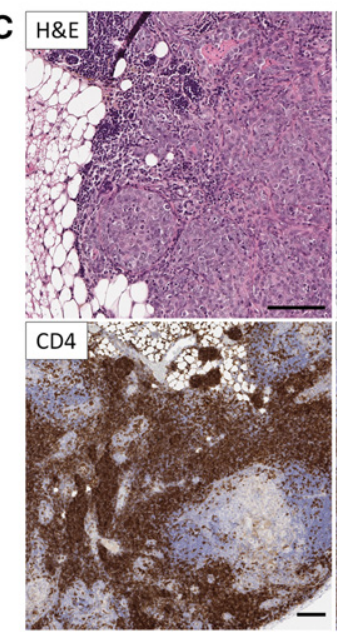

D

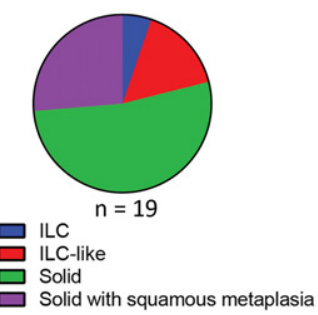

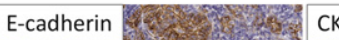

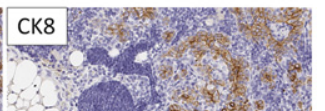

Figure 3. Intraductal injection of pSECC-sgPten in $C d h 1^{F / F}$ mice results in non-ILC tumors with strong immune infiltrations. (A) Analysis of pSECC-sgPten transduced Cre reporter cells $5 \mathrm{~d}$ after transduction. Percentage of GFP-positive cells and the spectrum of insertions/deletions (indels) of the targeted Pten alleles are shown by FACS and TIDE (tracking of indels by decomposition) analysis, respectively. The fraction of unmodified alleles is depicted in pink, while red bars $(P<0.001)$ and black bars $(P>0.001)$ represent the fractions of modified alleles. $(B) \mathrm{H} \& \mathrm{E}$ staining of a representative tumor section from $C d h 1^{F / F} ; A k t-E 17 K$ mice injected with pSECC-sgNT. $n=4$. Tumors were analyzed $16 \mathrm{wk}$ after injection. Bar, $100 \mu \mathrm{m} .(C)$ Immunohistochemical detection of E-cadherin-, CK8-, CD4-, CD8-, and B220expressing cells in tumor sections from $C d h 1^{F / F}$ mice injected with pSECC-sgPten. Tumor lesions were analyzed $25 \mathrm{wk}$ after injection. Bars, $100 \mu \mathrm{m}$. $(D)$ Histological classification of tumors from $C d h 1^{F / F}$ mice injected with pSECC-sgPten. $n=48$. ILC-like classification refers to lesions that are too small to display the typical ILC histological phenotype with the characteristic growth pattern. (E) TIDE analysis of the targeted Pten alleles in a representative tumor from pSECC-sgPten-injected $C d h 1^{F / F}$ mice.

S6A,D). Together, these data show that pSECC-mediated somatic Cre delivery and inactivation of Pten in mammary epithelial cells of $C d h 1^{F / F}$ mice induce tumors that do not resemble ILC and show a massive immune infiltration. Importantly, Lenti-Cre-mediated inactivation of Pten and E-cadherin in mammary glands of $C d h 1^{F / F} ;$ Pten $^{F / F}$ mice did not elicit a strong immune influx, suggesting that the immune infiltration in tumors induced by pSECC is not due to somatic Cre expression (Supplemental Fig. S4A).
Somatic gene editing and ILC formation in conditional Cas9 knock-in mice

Given that Cas9 was reported to be immunogenic (Wang et al. 2015), we hypothesized that Cas9-specific immune responses might have limited the success of pSECCsgPten for ILC modeling in $C d h 1^{F / F}$ female mice. We therefore generated $C d h 1^{F / F}$;Col1a1 $1^{\text {invCAG-Cas9-IRES-Luc/+ }}$ $\left(\mathrm{Cdh} 1^{F / F}\right.$;Cas 9$)$ mice with a Cre conditional Cas 9 allele in the Colla1 locus, as described above for the Akt-

Table 1. Overview of intraductal injections performed with pSECC and LentiGuide vectors, with affected mammary glands for each genotype

\begin{tabular}{|c|c|c|c|c|c|c|}
\hline Genotype & Vector & sgRNA & $\begin{array}{l}\text { Number of } \\
\text { injected mice }\end{array}$ & $\begin{array}{c}\text { Number of } \\
\text { injected glands }\end{array}$ & $\begin{array}{l}\text { Number of } \\
\text { affected glands }\end{array}$ & $\begin{array}{l}\text { Percentage of } \\
\text { affected glands }\end{array}$ \\
\hline$C d h 1^{F / F}$ & pSECC & sgPten & 26 & 48 & 12 & $25 \%$ \\
\hline$C d h 1^{F / F}$ & pSECC & sgNT & 14 & 27 & 0 & $0 \%$ \\
\hline WapCre;Cdh1 ${ }^{F / F}$;Cas9 & LentiGuide & sgPten & 14 & 27 & 8 & $30 \%$ \\
\hline WapCre;Cdh1 ${ }^{\text {F/F }}$;Cas9 & LentiGuide & sgNT & 8 & 14 & 0 & $0 \%$ \\
\hline WapCre;Cdh1 $1^{\text {F/F }}$ & LentiGuide & sgPten & 1 & 2 & 0 & $0 \%$ \\
\hline Cdh1 $1^{F / F}$;Cas 9 & LentiGuide & sgPten & 1 & 2 & 0 & $0 \%$ \\
\hline
\end{tabular}


E17K mutant (Supplemental Fig. S7A,B). Expression of Cre in mouse mammary epithelial cells (MMECs) derived from $C d h 1^{F / F}$; Cas 9 mice induced the inversion of the CAG promoter and subsequent Cas9 protein expression (Fig. 4A; Supplemental Fig. S7C). To test the genomic editing capacity of the conditional Cas 9 knock-in allele, we

A Adeno-Cre in $C d h 1^{F / F} ;$ Cas9 in vitro B B Lenti-Cre + LentiGuide-sgPten in vitro

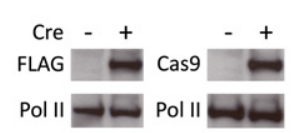
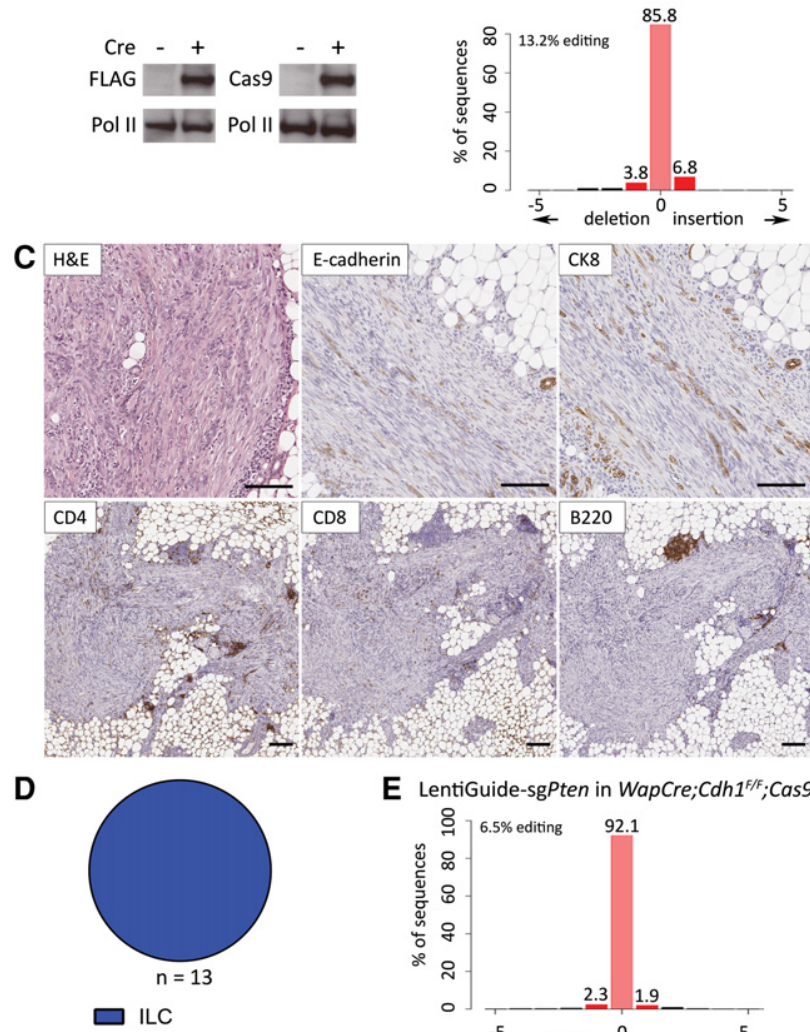

$\square$ ILC

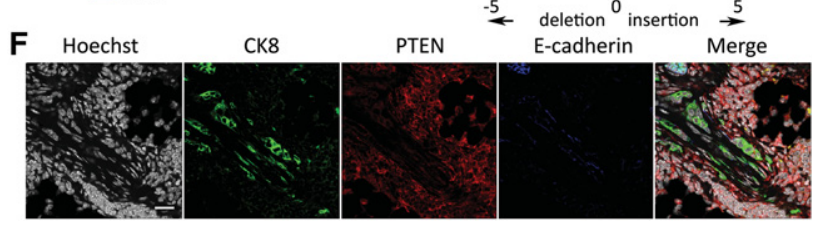

Figure 4. ILC formation in WapCre; $C d h 1^{F / F}$;Cas 9 mice injected with LentiGuide-sgPten. (A) Expression of Cas9 from the Cre conditional Cas 9 allele following in vitro transduction of $C d h 1^{F / F}$; Cas9 MMECs with Adeno-Cre, as visualized by immunoblotting with anti-Flag and anti-Cas9 antibodies. Pol II is shown as loading control. (B) TIDE analysis of the targeted Pten alleles in $C d h 1^{F / F}$; Cas9 MMECs cotransduced with Lenti-Cre and LentiGuidesgPten. (C) Immunohistochemical detection of E-cadherin-, CK8-, CD4-, CD8-, and B220-expressing cells in tumor sections from WapCre; $\operatorname{Cdh}^{F / F}$;Cas 9 mice injected with LentiGuidesgPten. Tumor lesions were analyzed 25 wk after injection. Bars, $100 \mu \mathrm{m}$. (D) Histological classification of tumors from WapCre; $C d h 1^{F / F}$;Cas 9 mice injected with LentiGuide-sgPten. $n=27$. (E) TIDE analysis of the targeted Pten alleles in a representative tumor from LentiGuide-sgPten-injected WapCre;Cdh1 $1^{F / F}$; Cas $9^{F / F}$ mice. $(F)$ Representative immunofluorescence imaging of tumor sections from LentiGuide-sgPten-injected WapCre; Cdh1 ${ }^{F / F}$;Cas 9 mice, stained with antibodies against CK8, PTEN, and E-cadherin. Bar, $25 \mu \mathrm{m}$. made use of a lentiviral vector (LentiGuide) encoding only sgPten or sgNT. Cotransduction of Lenti-Cre and LentiGuide-sgPten vectors in MMECs derived from $C d h 1^{F / F}$;Cas9 mice resulted in Pten gene editing in a fraction of cells (Fig. 4B). No insertions/deletions (indels) at the targeted location were observed upon cotransduction of Lenti-Cre and LentiGuide-sgNT or upon transduction with LentiGuide-sgPten alone, thus validating the functionality of the conditional Cas9 allele (Supplemental Fig. S7D). To determine the utility of this approach for ILC modeling, we performed intraductal injections with LentiGuide-sgPten $(n=27)$ or LentiGuide-sgNT $(n=14)$ in WapCre;Cdh1 $1^{F / F}$;Cas9 female mice, which were analyzed for ILC development 25 wk after injection. $C d h 1^{F / F}$;Cas9 or WapCre;Cdh1 ${ }^{F / F}$ control mice injected with LentiGuide-sgPten or WapCre;Cdh1 $1^{F / F}$;Cas9 mice injected with LentiGuide-sgNT did not display mammary tumor formation. In contrast, eight out of 27 WapCre; Cdh1 $1^{F / F}$;Cas 9 mammary glands injected with LentiGuide-sgPten developed one or more ILCs (Table 1; Supplemental Fig. S8A,B). All tumors showed typical ILC histology, characterized by discohesive CK8-positive and E-cadherin-negative tumor cells and an abundant presence of fibroblasts and collagen deposition (Fig. 4C,D). Moreover, tumors showed an extent of immune infiltration that was more limited than observed in the pSECCsgPten-induced tumors and comparable with ILCs from Lenti-Cre-injected $C d h 1^{F / F}{ }_{;} P t e n^{F / F}$ mice (Fig. 4C). These data suggest that WapCre;Cdh1 ${ }^{F / F}$;Cas 9 mice show immunological tolerance to WapCre-driven Cas9 expression in the mammary epithelium. This tolerance is likely caused by ectopic expression of Cas9 during the early stages of postnatal development, induced by WapCre activity in the brain (Supplemental Fig. S9A-D; Wagner et al. 1997). Target modification of Pten exon 1 was observed in genomic DNA from tumor-bearing mammary glands, and indels were exclusively frameshift mutations (Fig. 4E; Supplemental Fig. S8C). Consistent with this, tumors showed recombined $C d h 1^{F}$ and invCAG-Cas9IRES-Luc alleles; were positive for CK8 and negative for PTEN, E-cadherin, and vimentin; and showed activation of PI3K/AKT signaling (Fig. 4F; Supplemental Fig. S8D, E). Collectively, these data show that intraductal delivery of sgRNA-Pten in WapCre;Cdh1 ${ }^{F / F}$;Cas 9 female mice induces ILCs that closely resemble tumors from WapCre;Cdh1 ${ }^{F / F}{ }_{\text {;Pten }}{ }^{F / F}$ mice or Lenti-Cre injected $C d h 1^{F / F}$; $P_{t e n}{ }^{F / F}$ mice. Moreover, preliminary data suggest that intraductal injection of WapCre; $C d h 1^{F / F}$;Cas 9 mice with a single lentiviral vector encoding two sgRNAs may allow for multiplex gene editing, which would enable in vivo validation of combinations of TSGs implicated in ILC (Supplemental Fig. S10A-C).

\section{Discussion}

In this study, we describe novel approaches for nongermline modeling of E-cadherin-deficient lobular breast carcinoma by the delivery of lentiviral vectors via intraductal injection in the nipples of adult female mice. By using 
high-titer lentiviral vector preparations, we achieved extensive transduction of the ductal system and in vivo Cre-mediated recombination when using Lenti-Cre preparations in $m T / m G$ Cre reporter mice. This recapitulates previous studies using adenoviral vectors for somatic Cre delivery to murine mammary tissue (Rutkowski et al. 2014; Tao et al. 2014). We observed that the target cell population was composed of both CK8-positive luminal epithelial cells and CK14-positive basal cells. Intraductal administration of Lenti-Cre in the novel $C d h 1^{F / F} ; A k t$ $E 17 \mathrm{~K}$ and $C d h 1^{F / F}$;Pten ${ }^{F / F}$ mouse models resulted in the transduction of ILC-initiating cells, as shown by the highly penetrant and rapid ILC development in these animals. Tumors developing in these mice were histologically indistinguishable from those arising in the WapCre-based ILC models, suggesting that the cells targeted by intraductally injected lentiviruses are the same as the tumor-initiating cells in the spontaneous mouse models. Compared with the WapCre-driven model, Lenti-Cre injection simplifies breeding of experimental animals by eliminating the necessity of a Cre allele and allows a more sparse and stochastic targeting of ILC-initiating cells, better reflecting the sporadic nature of human cancer. Moreover, it allows spatiotemporal control of ILC initiation and permits study of the initiating events of ILC in the adult mammary gland, whereas WapCre is already active during prepuberal developmental stages. Furthermore, while transgenic animals often develop mammary tumors in multiple glands, tumor induction by intraductal injection can be restricted to a single gland, yielding de novo mammary tumor models suitable for studying the development of metastatic disease following removal of the primary tumor and evaluating the efficacy of adjuvant systemic therapies.

A possible limitation of intraductal Cre delivery is the inherent lack of specificity of viral transduction, which might also target non-ILC-initiating cells in the mammary gland. Nonetheless, this promiscuity might be advantageous in case the cell of origin is unknown and might enable modeling of other breast cancer subtypes by intraductal administration of Lenti-Cre to mice bearing different predisposing mutations, provided that the cells of origin for that tumor type can be transduced. Additionally, viral vectors in which Cre recombinase expression is driven by tissue-specific promoters might be used to target specific subtypes of mammary epithelial cells (Tao et al. 2014).

Although intraductal injection of Lenti-Cre provides an effective approach to model breast cancer in mice, its applicability is dependent on the generation of effective conditional alleles for relevant oncogenes and tumor suppressors and their incorporation in compound mice. An alternative approach that enables more rapid and methodic in vivo gene editing would be required to functionally validate the increasing numbers of candidate driver mutations that are being identified by genomewide sequencing studies of human breast cancer (The Cancer Genome Atlas Network 2012; Stephens et al. 2012; Ciriello et al. 2015). The advent of CRISPR/Cas9 technologies allows for rapid somatic gene editing in nearly any cell type to study the effects of gene perturbation in situ. Several studies have already shown that tumor initiation and development in various tissues, including the liver, lung, pancreas, and brain, can be modeled in vivo by using CRISPR/Cas9-based somatic gene editing (Platt et al. 2014; Sánchez-Rivera et al. 2014; Xue et al. 2014; Chiou et al. 2015; Weber et al. 2015; Zuckermann et al. 2015). As a proof of concept, we performed intraductal injections with pSECC-sgPten lentiviral vectors in $C d h 1^{F / F}$ female mice to simultaneously ablate Ecadherin expression and disrupt the TSG Pten, a negative regulator of $\mathrm{PI} 3 \mathrm{~K} / \mathrm{AKT}$ signaling. While tumor lesions were observed in a limited number of animals, they did not resemble ILC and showed incomplete loss of E-cadherin, suggesting that tumorigenesis in $C d h 1^{F / F}$ mice injected with pSECC-sgPten is driven by PTEN loss rather than combined inactivation of both tumor suppressors. Moreover, all tumors in these mice showed a more profound immune infiltration than the ILCs arising in Lenti-Cre-injected $C d h 1^{F / F}{ }_{\text {Pten }}{ }^{F / F}$ animals, which might be due to humoral and cellular immunity against $S$. pyogenes Cas9 (Wang et al. 2015), an aspect that thus far has been underappreciated in in vivo CRISPR/Cas9 studies. To avoid Cas9-directed immunity, we performed intraductal injections of LentiGuide-sgPten in WapCre; $C d h 1^{F / F}$; Cas9 female mice, which express the Cas9 endonuclease from a conditional knock-in allele in the mammary epithelium. This resulted in E-cadherin-negative tumor lesions resembling human ILC in $30 \%$ of the injected mammary glands upon a single administration of the lentiviral vector. Incomplete tumor penetrance could reflect reduced number of ILC-initiating cells in WapCre;Cdh $1^{F / F}$;Cas 9 female mice compared with wild-type mice, which might be due to the fact that E-cadherin loss in mammary epithelial cells induces apoptosis (Boussadia et al. 2002). The lack of a massive immune infiltration in these tumors indicates that conditional Cas9 expression in the brain during early postnatal development leads to tolerance in WapCre; $C d h 1^{F / F}$; Cas 9 mice, resulting in efficient ILC development following CRISPR/Cas9-mediated disruption of the Pten alleles. Indeed, TIDE (tracking of indels by decomposition) analyses of tumor-bearing mammary glands exclusively showed frameshifting genetic alterations in Pten and concomitant activation of phospho-AKT ${ }^{\text {Ser473, }}$, indicating positive selection for cells with disrupted PTEN in E-cadherin-deficient cells and providing functional support for the notion that activating mutations in the PI3K/AKT signaling pathway and inactivating mutations in CDH1 effectively collaborate in human ILC development.

Taken together, we show for the first time that CRISPR/ Cas9-mediated somatic gene editing of mammary epithelial cells can be used to target and genetically modify ILCinitiating cells by intraductal injection of sgRNA-encoding lentiviral vectors in WapCre; $C d h 1^{F / F}$; Cas9 female mice. This approach allows rapid in vivo testing of putative co-occurring mutations with E-cadherin loss to initiate invasive lobular breast carcinoma and, in principle, could be extended to other breast cancer subtypes. Our 
preliminary data with single lentiviral vectors encoding multiple sgRNAs suggest that WapCre;Cdh1 ${ }^{F / F}$;Cas 9 mice may be used for multiplex gene editing of the mammary gland in order to test combinations of TSGs implicated in ILC. It will be interesting to determine whether WapCre;Cdh1 ${ }^{F / F}$;Cas 9 mice may also be used for in vivo forward genetic screens with focused CRISPR libraries to identify novel TSGs critical for ILC development. To test candidate drivers that are overexpressed or amplified in ILC, it may be relevant to develop WapCre; $C d h 1^{F / F}$; dCas9-p300core mice with conditional expression of nuclease-deficient Cas9 fused to the catalytic core of the human acetyltransferase p300 (Hilton et al. 2015).

\section{Materials and methods}

\section{Lentiviral vectors}

The LentiGuide vector was a kind gift from Feng Zhang (Addgene, plasmid no. 52963). The pSECC vector was a kind gift from Tyler Jacks (Addgene, plasmid no. 60820). The sgRNA targeting Pten exon 1 (GCTAACGATCTCTTTGATGA) was the validated gRNA used by Sánchez-Rivera et al. (2014), while the nontargeting gRNA (TGATTGGGGGTCGTTCGCCA) was selected from the list of nontargeting gRNAs of the GeCKO version 2 mouse gRNA library (Sanjana et al. 2014). Cloning of gRNAs in LentiGuide and pSECC was performed as described (Sanjana et al. 2014). Tandem sgRNA vectors were made by cloning in tandem either two sgNT expression cassettes or the Pten gRNA expression cassette followed by a gRNA expression cassette targeting $\operatorname{Trp} 53$ exon 5 (GAAGTCACAGCACATGACGG) (data not shown). All vectors were validated by Sanger sequencing. LentiCre (pBOB-CAG-iCRE-SD; Addgene, plasmid no. 12336) was a kind gift of Lorenzo Bombardelli. We produced concentrated lentiviral stocks, pseudotyped with the VSV-G envelope, by transient cotransfection of four plasmids in 293T cells as previously described (Follenzi et al. 2000). Viral titers were determined using the quantitative PCR (qPCR) lentivirus titration kit from Abm (LV900).

\section{Cell culture}

MMECs were isolated from 12-wk-old females as previously described (Ewald et al. 2008) and cultured in DMEM-F12 medium containing $10 \%$ fetal bovine serum (FBS), $100 \mathrm{IU} / \mathrm{mL}$ penicillin, $100 \mu \mathrm{g} / \mathrm{mL}$ streptomycin, $5 \mathrm{ng} / \mathrm{mL}$ insulin, $5 \mathrm{ng} / \mathrm{mL}$ epidermal growth factor (EGF) (all from Life Technologies), and $5 \mathrm{ng} / \mathrm{mL}$ cholera toxin (Sigma). 293T cells for lentiviral production and the Cre reporter $293 \mathrm{~T}$ cell line (containing a lox-stop-lox-GFP cassette) were cultured in Iscove's medium (Life Technologies) containing $10 \%$ FBS, $100 \mathrm{IU} / \mathrm{mL}$ penicillin, and $100 \mu \mathrm{g} / \mathrm{mL}$ streptomycin. Transductions were performed by adding diluted viral supernatant to the cells in the presence of $8 \mu \mathrm{g} / \mathrm{mL}$ polybrene (Sigma). Cells were transduced at a multiplicity of infection (MOI) of 10 for $24 \mathrm{~h}$, after which the medium was refreshed. Harvesting of cells for flow cytometry and/or genomic DNA isolation was performed $5 \mathrm{~d}$ after transduction.

\section{Flow cytometry}

Cells were collected $5 \mathrm{~d}$ after transduction and directly analyzed for GFP fluorescence using a Becton Dickinson FACSCalibur.
Viable cells were gated on size and shape using forward and side scatter. GFP expression was measured using a 488-nm excitation laser. Data analysis was performed using FlowJo software version 7.6.5.

Genomic DNA isolation, PCR amplification, and TIDE analysis

Genomic DNA from frozen cell pellets and mammary gland frozen pieces was isolated using the Gentra Puregene genomic DNA isolation kit from Qiagen. PCR amplification of Pten exon 1 or Trp53 exon 5 was performed with specific primers spanning the target site (FW_Pten, GCCCAGTCTCTGCAACC ATC; RV_Pten, CACGATCTAGAAATGCGCCC; FW_Trp53, CCCACCTTGACACCTGATCG; and RV_Trp53, CCACCCGG ATAAGATGCTGG) and $1 \mu \mathrm{g}$ of DNA template using the Q5 high-fidelity PCR kit from New England Biolabs. Amplicons were run on $1 \%$ agarose gel, and gel purification was performed using the Isolate II PCR and gel kit from Bioline. PCR products were Sanger-sequenced using the FW primer, and CRISPR/ Cas9-induced editing efficacy was quantified with the TIDE algorithm as described (Brinkman et al. 2014; http://tide.nki.nl). Nontransduced cells were used as a negative control in all genomic DNA amplifications, and only TIDE outputs with $R^{2}>0.9$ were considered. Inversion of the CAG promoter (Huijbers et al. 2015) of the Akt-E17K conditional allele was detected as described (Huijbers et al. 2014) with a shared FW primer located on Lox66 (primer 1, GGCCGGCCATAACTTCGTATAATG) and two RV primers-one located in the vector backbone (primer 2, CTGCGTTATCCCCTGATTCTGTGG) to detect the nonrecombined allele (product size 897 base pairs [bp]) and one located in the hygromycin-B resistance gene (primer 3, CCTACATC GAAGCTGAAAGCACGAG) to identify the recombined allele (product size $1054 \mathrm{bp}$ ). Inversion of the CAG promoter of the Cas 9 conditional allele was detected using the Q5 kit to amplify the Col1a1 locus with a shared FW primer located on the CAG promoter itself (primer 1, CTTCTCCCTCTCCAGCCTCGGG) and two RV primers-one located on the hygromycin-B resistance gene (primer 2, CATCAGGTCGGAGACGCTGTCG) and one located on the Cas9 shuttle (primer 3, TCGACGGA TCTTGGGAGGCCTA). PCR amplification with primers 1 and 2 identified a band of 386 bp: the nonrecombined shuttle construct. PCR amplification with primers 1 and 3 identifies a band of $264 \mathrm{bp}$ when Cre-mediated recombination of the shuttle construct had occurred. $C d h 1^{F}$ and $C d h 1^{\Delta}$ alleles were identified by PCR as described (Derksen et al. 2006). Pten ${ }^{F}$ alleles were detected by PCR using primers located in intron 5 (FW, TGGGGGTATTCACTAGTATAG; and RV, GAGTCCTCTG AAAAAGCAGTC; product size $200 \mathrm{bp}$. Pten $^{\Delta}$ alleles were detected using a FW primer in intron 4 (CCTAGGCTACTGCT CATT) and the RV primer located in intron 5 (product size 350 bp). The tandem vector was detected using the Q5 high-fidelity PCR kit from New England Biolabs. The FW primer was located at the human U6 promoter (primer 1, CAAAGATATTAGTA CAAAATACGT), and RV primer was located at the SFFV promoter (primer 2, TGAACTTCTCTATTCTTGGTTTGGT; product size $831 \mathrm{bp})$.

\section{Adeno-Cre transduction in vitro}

MMECs were seeded in six-well plates, and confluent wells were transduced with viral Ad5-CMV-Cre particles $\left(1 \times 10^{8}\right.$ transducing units [TU]; Gene Transfer Vector Core, University of Iowa) in the presence of $8 \mu \mathrm{g} / \mathrm{mL}$ polybrene (Sigma). Five days after transduction, DNA and proteins were isolated. 


\section{Immunoblotting}

Protein lysates were made using lysis buffer $(20 \mathrm{mM}$ Tris at $\mathrm{pH}$ 8.0, $300 \mathrm{mM} \mathrm{NaCl}, 2 \%$ NP40, 20\% glycerol, $10 \mathrm{mM}$ EDTA) complemented with protease inhibitors (Roche) and quantified using the BCA protein assay kit (Pierce). Protein lysate was loaded onto a 3\%-8\% Tris-acetate gradient gel (Invitrogen) and transferred overnight onto PVDF membrane (Millipore) in transfer buffer (238 mM glycine, $80 \mathrm{mM}$ TRIS, $0.01 \%$ SDS in water). Membranes were blocked in 5\% ELK in PBS-T $10.05 \%$ Tween$20)$, after which they were stained for $4 \mathrm{~h}$ at room temperature using the primary antibodies anti-Flag (1:1000; Sigma Aldrich, F1804) and anti-Cas9 (1:1000; Cell Signaling, 14697) in 1\% ELK in PBS-T. Pol II (1:400; Santa Cruz Biotechnology, sc-5943) was incubated for $1 \mathrm{~h}$ at room temperature in $1 \%$ ELK in PBS-T. Membranes were washed three times with $1 \%$ ELK in PBS-T and incubated for $1 \mathrm{~h}$ with an HRP-conjugated secondary antibody 1 1:2000; DAKO). Stained membranes were washed three times in $1 \%$ ELK in PBS-T and then developed using SuperSignal ECL (Pierce).

\section{Mice}

Akt1 cDNA (Open Biosystems, 100067520) was modified using site-directed mutagenesis (Agilent QuikChange Lightning multikit; FW, GCTGCACAAACGAGGGAAGTACATCAAGACC TG; and RV, CAGGTCTTGATGTACTTCCCTCGTTTGTGC AGC), resulting in mutant Akt-E17K. Akt-E17K and Cas9 (Addgene, plasmid no. 42229) cDNAs were sequence-verified and inserted as FseI-PmeI and BamHI fragments, respectively, into the Frt-invCag-IRES-Luc vector, resulting in Frt-invCagAktE17K-IRES-Luc and Frt-invCag-Cas9-IRES-Luc. Flp-mediated integration of the shuttle vectors in WapCre;Cdh1 $1^{F / F}$; Colla1 $1^{f r t /+}$ GEMM-ESC clones was performed as described (Huijbers et al. 2015). Chimeric animals were crossed with WapCre; $C d h 1^{F / F}$ and $C d h 1^{F / F}$ animals to generate the cohorts. WapCre, Cdh1 $1^{F}, \mathrm{mT} / \mathrm{mG}$, Col1a1 invCAG-AktE17K-IRES-Luc, and

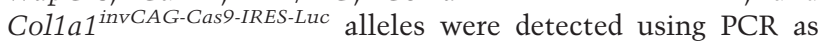
described (Derksen et al. 2006, 2011; Muzumdar et al. 2007; Huijbers et al. 2014). Pten ${ }^{F}$ alleles were detected by standard PCR at an annealing temperature of $58^{\circ} \mathrm{C}$ using primers located in intron 5 (FW, TGGGGGTATTCACTAGTATAG; and RV, GAGTCCTCTGAAAAAGCAGTC; product size 200 bp) (Marino et al. 2002).

\section{In vivo bioluminescence imaging}

In vivo bioluminescence imaging was performed as described (Henneman et al. 2015) by using a cooled CCD system (Xenogen Corp.) coupled to Living Image acquisition and analysis software (Xenogen). Signal intensity was measured over the region of interest and quantified as flux (photons per second per square centimeter per steradian).

\section{Intraductal injections}

Intraductal injections were performed as described (Krause et al. 2013). Briefly, the mice were anesthetized using $100 \mathrm{mg} / \mathrm{kg}$ ketamine and $10 \mathrm{mg} / \mathrm{kg}$ sedazine, and hair was removed in the nipple area with a commercial hair removal cream. Eighteen microliters of high-titer lentivirus (or adenovirus) mixed with $2 \mu \mathrm{L}$ of $0.2 \%$ Evans blue dye in PBS was injected in the fourth mammary gland using a 34-gauge needle. Mice were handled in a biological safety cabinet under a stereoscope. Lentiviral titers ranging from $2 \times 10^{8}$ $\mathrm{TU} / \mathrm{mL}$ to $2 \times 10^{9} \mathrm{TU} / \mathrm{mL}$ were used. Animal experiments were approved by the Animal Ethics Committee of the Netherlands
Cancer Institute and performed in accordance with institutional, national, and European guidelines for animal care and use.

\section{Fluorescence imaging of freshly isolated tissue}

FVB mice injected with Lenti-GFP, $m T / m G$ animals injected with Lenti-Cre, and $m T / m G$ mice injected with Adeno-Cre were sacrificed $3 \mathrm{~d}$ or $2 \mathrm{wk}$ after injection. Mammary glands were isolated and kept in PBS on ice prior to imaging. The brains and the mammary glands of the WapCre; $m T / m G$ pups were isolated at the indicated time points. Images were acquired by using a Zeiss AxioZoom.V16 stereo microscope and analyzed by ZEN lite 2012 (Blue edition) software.

\section{Histology and immunohistochemistry}

Tissues were formalin-fixed and paraffin-embedded by routine procedures. H\&E staining was performed as described (Doornebal et al. 2013). Five semiserial slides per injected mammary gland were stained with $\mathrm{H} \& \mathrm{E}$ and reviewed by a blinded and dedicated pathologist (S. Klarenbeek) according to international consensus of mammary pathology (Cardiff et al. 2000). Quantitation of the number of tumors per gland was performed using a single H\&Estained slide per mammary gland. Tumor burden was calculated as the ratio between the total tumor area and the area of the whole mammary gland using ImageJ software version 1.4.3.67. Immunohistochemical stainings were processed as described (Doornebal et al. 2013; Henneman et al. 2015). Antibody details and antigen retrieval methods are described in Supplemental Table S1. All slides were digitally processed using the Aperio ScanScope (Aperio) and captured using ImageScope software version 12.0.0 (Aperio).

\section{Immunofluorescence}

Formalin-fixed and paraffin-embedded sections were processed as described (Pasic et al. 2011). Sections were incubated overnight at $4^{\circ} \mathrm{C}$ with primary antibodies anti-CK8 $(1: 100$; University of Iowa, TROMA-1), anti-CK14 (1:200; Covance, PRB-155P), anti-PTEN (1:100; Cell Signaling, 9188), and anti-E-cadherin (1:100; BD Biosciences, 610181). Secondary antibodies anti-rat Alexa fluor 647 (1:1000; Invitrogen, A21247), anti-rabbit Alexa fluor 568 (1:1000; Invitrogen, A11011), and anti-mouse Alexa fluor 488 (1:1000; Molecular Probes, A21141) were incubated overnight at $4^{\circ} \mathrm{C}$. Sections were subsequently stained with Hoechst 1:1000; Thermo Scientific, 62249) for $5 \mathrm{~min}$ and mounted using VectaShield (Vector Laboratories, H-1000). Images were acquired using a Leica TCS SP5 confocal microscope and analyzed using LAS AF version 2.6.3 software.

\section{Acknowledgments}

We are grateful to Marco Barazas, Chiara Brambillasca, Bastiaan Evers, Francisco J. Sánchez-Rivera, Tyler Jacks, Lorenzo Bombardelli, Ingrid van der Heijden, Ellen Wientjens, Renske de KorteGrimmerink, and Natalie Proost for providing valuable reagents, technical suggestions, and/or help with the experiments, and Jelle Wesseling for critical reading of the manuscript. We thank the Netherlands Cancer Institute Animal Facility, Animal Pathology Facility, Core Facility Molecular Pathology and Biobanking (CFMPB), Flow Cytometry Facility, and Genomics Core Facility for their expert technical support. Financial support was provided by the Netherlands Organization for Scientific Research (NWO; Cancer Genomics Netherlands [CGCNL] and Cancer Systems 
Biology Center [CSBC] VENI 016156012 to M.N., and Netherlands Genomics Initiative Zenith 93512009 and VICI 91814643 to J.J.), Worldwide Cancer Research (grant 14-0288 to J.J. and M. H.v.M.), the European Union Seventh Framework Program (EurocanPlatform project 260791 and Infrafrontier-I3 project 312325 ), the European Research Council (ERC Synergy project CombatCancer), and a National Roadmap grant for Large-Scale Research Facilities from the NWO.

\section{References}

Borst MJ, Ingold JA. 1993. Metastatic patterns of invasive lobular versus invasive ductal carcinoma of the breast. Surgery 114: 637-641.

Boussadia O, Kutsch S, Hierholzer A, Delmas V, Kemler R. 2002. $\mathrm{E}$-cadherin is a survival factor for the lactating mouse mammary gland. Mech Dev 115: 53-62.

Brinkman EK, Chen T, Amendola M, van Steensel B. 2014. Easy quantitative assessment of genome editing by sequence trace decomposition. Nucleic Acids Res 42: 168.

The Cancer Genome Atlas Network. 2012. Comprehensive molecular portraits of human breast tumours. Nature 490: 61-70.

Cardiff RD, Anver MR, Gusterson BA, Hennighausen L, Jensen RA, Merino MJ, Rehm S, Russo J, Tavassoli FA, Wakefield LM, et al. 2000. The mammary pathology of genetically engineered mice: the consensus report and recommendations from the Annapolis meeting. Oncogene 19: 968-988.

Chiou S-H, Winters IP, Wang J, Naranjo S, Dudgeon C, Tamburini FB, Brady JJ, Yang D, Grüner BM, Chuang C-H, et al. 2015. Pancreatic cancer modeling using retrograde viral vector delivery and in vivo CRISPR/Cas9-mediated somatic genome editing. Genes Dev 29: 1576-1585.

Ciriello G, Gatza ML, Beck AH, Wilkerson MD, Rhie SK, Pastore A, Zhang H, McLellan M, Yau C, Kandoth C, et al. 2015. Comprehensive molecular portraits of invasive lobular breast cancer. Cell 163: 506-519.

Derksen PWB, Liu X, Saridin F, van der Gulden H, Zevenhoven I, Evers B, van Beijnum JR, Griffioen AW, Vink J, Krimpenfort P, et al. 2006. Somatic inactivation of E-cadherin and p53 in mice leads to metastatic lobular mammary carcinoma through induction of anoikis resistance and angiogenesis. Cancer Cell 10: 437-449.

Derksen PWB, Braumuller TM, van der Burg E, Hornsveld M, Mesman E, Wesseling J, Krimpenfort P, Jonkers J. 2011. Mammary-specific inactivation of E-cadherin and p53 impairs functional gland development and leads to pleomorphic invasive lobular carcinoma in mice. Dis Model Mech 4: 347-358.

Doornebal CW, Klarenbeek S, Braumuller TM, Klijn CN, Ciampricotti M, Hau C-S, Hollmann MW, Jonkers J, de Visser KE. 2013. A preclinical mouse model of invasive lobular breast cancer metastasis. Cancer Res 73: 353-363.

Droufakou S, Deshmane V, Roylance R, Hanby A, Tomlinson I, Hart IR. 2001. Multiple ways of silencing E-cadherin gene expression in lobular carcinoma of the breast. Int I Cancer 92: 404-408.

Ewald AJ, Brenot A, Duong M, Chan BS, Werb Z. 2008. Collective epithelial migration and cell rearrangements drive mammary branching morphogenesis. Dev Cell 14: 570-581.

Follenzi A, Ailles LE, Bakovic S, Geuna M, Naldini L. 2000. Gene transfer by lentiviral vectors is limited by nuclear translocation and rescued by HIV-1 pol sequences. Nat Genet 25: 217-222.
Harada N, Oshima H, Katoh M, Tamai Y, Oshima M, Taketo MM. 2004. Hepatocarcinogenesis in mice with $\beta$-catenin and Ha-ras gene mutations. Cancer Res 64: 48-54.

Henneman L, van Miltenburg MH, Michalak EM, Braumuller TM, Jaspers JE, Drenth AP, de Korte-Grimmerink R, Gogola E, Szuhai K, Schlicker A, et al. 2015. Selective resistance to the PARP inhibitor olaparib in a mouse model for BRCA1deficient metaplastic breast cancer. Proc Natl Acad Sci 112: 8409-8414.

Hilton IB, D'Ippolito AM, Vockley CM, Thakore PI, Crawford GE, Reddy TE, Gersbach CA. 2015. Epigenome editing by a CRISPR-Cas9-based acetyltransferase activates genes from promoters and enhancers. Nat Biotechnol 33: 510-517.

Howell JM, Lochmüller H, O'Hara A, Fletcher S, Kakulas BA, Massie B, Nalbantoglu J, Karpati G. 1998. High-level dystrophin expression after adenovirus-mediated dystrophin minigene transfer to skeletal muscle of dystrophic dogs: prolongation of expression with immunosuppression. Hum Gene Ther 9: 629-634.

Huijbers IJ, Bin Ali R, Pritchard C, Cozijnsen M, Kwon M-C, Proost N, Song J-Y, de Vries H, Badhai J, Sutherland K, et al. 2014. Rapid target gene validation in complex cancer mouse models using re-derived embryonic stem cells. EMBO Mol Med 6: 212-225.

Huijbers IJ, Del Bravo J, Bin Ali R, Pritchard C, Braumuller TM, van Miltenburg MH, Henneman L, Michalak EM, Berns A, Jonkers J. 2015. Using the GEMM-ESC strategy to study gene function in mouse models. Nat Protoc 10: 1755-1785.

Kirsch DG, Dinulescu DM, Miller JB, Grimm J, Santiago PM, Young NP, Nielsen GP, Quade BJ, Chaber CJ, Schultz CP, et al. 2007. A spatially and temporally restricted mouse model of soft tissue sarcoma. Nat Med 13: 992-997.

Krause S, Brock A, Ingber DE. 2013. Intraductal injection for localized drug delivery to the mouse mammary gland. $J$ Vis Exp. 80: 50692.

Marino S, Krimpenfort P, Leung C, van der Korput HAGM, Trapman J, Camenisch I, Berns A, Brandner S. 2002. PTEN is essential for cell migration but not for fate determination and tumourigenesis in the cerebellum. Development 129: 3513-3522.

Martinez V, Azzopardi JG. 1979. Invasive lobular carcinoma of the breast: incidence and variants. Histopathology 3: 467-488.

Meuwissen R, Linn SC, van der Valk M, Mooi WJ, Berns A. 2001. Mouse model for lung tumorigenesis through Cre/lox controlled sporadic activation of the K-Ras oncogene. Oncogene 20: 6551-6558.

Moll R, Mitze M, Frixen UH, Birchmeier W. 1993. Differential loss of E-cadherin expression in infiltrating ductal and lobular breast carcinomas. Am J Pathol 143: 1731-1742.

Muzumdar MD, Tasic B, Miyamichi K, Li L, Luo L. 2007. A global double-fluorescent Cre reporter mouse. Genesis 45: 593-605.

Pasic L, Eisinger-Mathason TSK, Velayudhan BT, Moskaluk CA, Brenin DR, Macara IG, Lannigan DA. 2011. Sustained activation of the HER1-ERK1/2-RSK signaling pathway controls myoepithelial cell fate in human mammary tissue. Genes Dev 25: 1641-1653.

Platt RJ, Chen S, Zhou Y, Yim MJ, Swiech L, Kempton HR, Dahlman JE, Parnas O, Eisenhaure TM, Jovanovic M, et al. 2014. CRISPR-Cas9 knockin mice for genome editing and cancer modeling. Cell 159: 440-455.

Rakha EA, Patel A, Powe DG, Benhasouna A, Green AR, Lambros MB, Reis-Filho JS, Ellis IO. 2010. Clinical and biological significance of E-cadherin protein expression in invasive lobular carcinoma of the breast. Am J Surg Pathol 34: 1472-1479. 
Russell TD, Fischer A, Beeman NE, Freed EF, Neville MC, Schaack J. 2003. Transduction of the mammary epithelium with adenovirus vectors in vivo. J Virol 77: 5801-5809.

Rutkowski MR, Allegrezza MJ, Svoronos N, Tesone AJ, Stephen TL, Perales-Puchalt A, Nguyen J, Zhang PJ, Fiering SN, Tchou J, et al. 2014. Initiation of metastatic breast carcinoma by targeting of the ductal epithelium with adenovirus-cre: a novel transgenic mouse model of breast cancer. J Vis Exp. 85: 51171.

Sánchez-Rivera FJ, Papagiannakopoulos T, Romero R, Tammela T, Bauer MR, Bhutkar A, Joshi NS, Subbaraj L, Bronson RT, Xue W, et al. 2014. Rapid modelling of cooperating genetic events in cancer through somatic genome editing. Nature 516: 428-431.

Sanjana NE, Shalem O, Zhang F. 2014. Improved vectors and genome-wide libraries for CRISPR screening. Nat Methods 11: 783-784.

Stephens PJ, Tarpey PS, Davies H, Van Loo P, Greenman C, Wedge DC, Nik-Zainal S, Martin S, Varela I, Bignell GR, et al. 2012. The landscape of cancer genes and mutational processes in breast cancer. Nature 486: 400-404.

Tao L, van Bragt MPA, Laudadio E, Li Z. 2014. Lineage tracing of mammary epithelial cells using cell-type-specific cre-expressing adenoviruses. Stem Cell Reports 2: 770-779.

Vos CB, Cleton-Jansen AM, Berx G, de Leeuw WJ, ter Haar NT, van Roy F, Cornelisse CJ, Peterse JL, van de Vijver MJ. 1997. E-cadherin inactivation in lobular carcinoma in situ of the breast: an early event in tumorigenesis. Br I Cancer 76: 1131-1133.
Wagner KU, Wall RJ, St-Onge L, Gruss P, Wynshaw-Boris A, Garrett L, Li M, Furth PA, Hennighausen L. 1997. Cre-mediated gene deletion in the mammary gland. Nucleic Acids Res 25: 4323-4330.

Wang D, Mou H, Li S, Li Y, Hough S, Tran K, Li J, Yin H, Anderson DG, Sontheimer EJ, et al. 2015. Adenovirus-mediated somatic genome editing of Pten by CRISPR/Cas9 in mouse liver in spite of Cas9-specific immune responses. Hum Gene Ther 26: 432-442.

Weber J, Öllinger R, Friedrich M, Ehmer U, Barenboim M, Steiger K, Heid I, Mueller S, Maresch R, Engleitner T, et al. 2015. CRISPR/Cas9 somatic multiplex-mutagenesis for highthroughput functional cancer genomics in mice. Proc Nat1 Acad Sci 112: 13982-13987.

Wong H, Lau S, Cheung P, Wong TT, Parker A, Yau T, Epstein RJ. 2014. Lobular breast cancers lack the inverse relationship between ER/PR status and cell growth rate characteristic of ductal cancers in two independent patient cohorts: implications for tumor biology and adjuvant therapy. BMC Cancer 14: 826.

Xue W, Chen S, Yin H, Tammela T, Papagiannakopoulos T, Joshi NS, Cai W, Yang G, Bronson R, Crowley DG, et al. 2014. CRISPR-mediated direct mutation of cancer genes in the mouse liver. Nature 514: 380-384.

Zuckermann M, Hovestadt V, Knobbe-Thomsen CB, Zapatka M, Northcott PA, Schramm K, Belic J, Jones DTW, Tschida B, Moriarity B, et al. 2015. Somatic CRISPR/Cas9-mediated tumour suppressor disruption enables versatile brain tumour modelling. Nat Commun 6: 7391. 


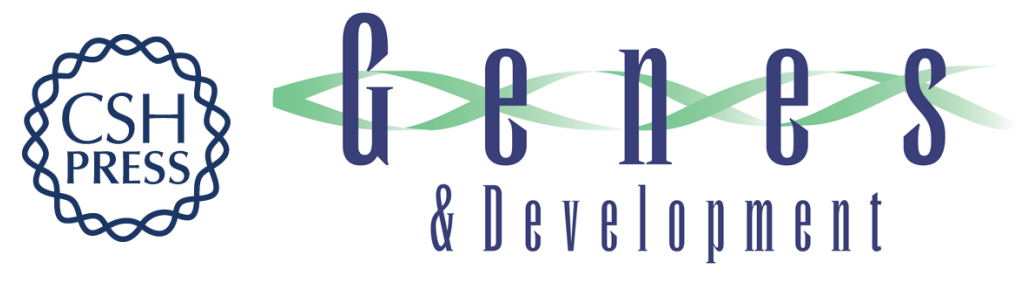

\section{Modeling invasive lobular breast carcinoma by CRISPR/Cas9-mediated somatic genome editing of the mammary gland}

Stefano Annunziato, Sjors M. Kas, Micha Nethe, et al.

Genes Dev. 2016, 30:

Access the most recent version at doi:10.1101/gad.279190.116

Supplemental http://genesdev.cshlp.org/content/suppl/2016/06/23/30.12.1470.DC1

Material

References This article cites 42 articles, 9 of which can be accessed free at: http://genesdev.cshlp.org/content/30/12/1470.full.html\#ref-list-1

Creative This article is distributed exclusively by Cold Spring Harbor Laboratory Press for the first Commons six months after the full-issue publication date (see

License http://genesdev.cshlp.org/site/misc/terms.xhtml). After six months, it is available under a Creative Commons License (Attribution-NonCommercial 4.0 International), as described at http://creativecommons.org/licenses/by-nc/4.0/.

Email Alerting Receive free email alerts when new articles cite this article - sign up in the box at the top Service right corner of the article or click here.

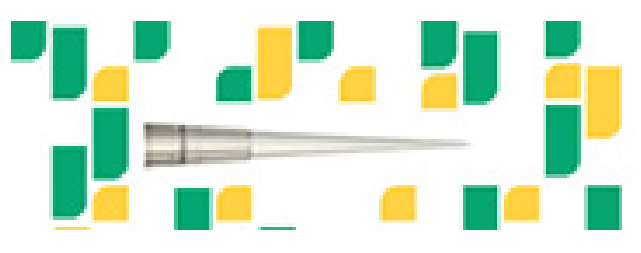

Focused on your science. 